\title{
NOUVELLES CONTRIBUTIONS A LA CONNAISANCE DE L'HISTOIRE DE LA VEGETATION HOLOCENE DES MONTS RODNEI (CARPATES ORIENTALES)
}

\author{
IOAN TANȚĂU 1
}

\begin{abstract}
New Contributions to the Knowledge of the Holocene Vegetation History of Rodnei Mountains. Two sequences originating from one peat bog in Poiana Ştiol (Rodnei Mts.) were pollen analyzed. The vegetation history is described since the Atlantic period. In this period the vegetation is dominated by Picea together with Corylus, Ulmus and Alnus viridis. The components frequencies of the Quercetum mixtum (Quercus, Fraxinus, Tilia, Corylus) rarely exceed 10\%. The vegetation of the Subboreal period is dominated by the Carpinus and that of the Subatlantic is dominated by Fagus. Picea pollen is well represented belong the entire sequences.
\end{abstract}

Key words. Palynology, Vegetation History, Holocene, Rodnei Mts., Romania.

\section{INTRODUCTION}

L'histoire de la végétation des Monts Rodnei est déjà bien connue grâce aux études palynologiques effectuées par Ciobanu et al. $(1969,1972)$ Boşcaiu et al. $(1983)$, Tanțău et Fărcaş (2002). Cet ouvrage vient de compléter les données palynologiques obtenues à Poiana Stiol par Tanțău (2003), Tanțău et Fărcaş (2004).

Monts Rodnei appartiennent au groupe nordique des Carpates Orientales (Fig. 1), étant les plus hautes montagnes de ce secteur.

Les traces de la glaciation quaternaire se retrouvent dans le relief actuel des Monts Rodnei: des cirques glaciaires qui abritent des lacs, des vallées glaciaires et des morènes. Les plus hauts sommets de ces montagnes sont Pietrosul Mare (2303 m) et Ineu $(2280 \mathrm{~m})$.

Le climat de la région est du à la position géographique des Monts Rodnei et à l'orientation ouest-est de la chaîne principale. Le type de climat est continentalmodéré, avec des faibles influences nord-atlantiques. Les températures moyennes annuelles varient en fonction de l'altitude et de l'exposition. Ainsi, au-dessus de $2200 \mathrm{~m}$ d'altitude la température moyenne est $-1,5^{\circ} \mathrm{C}$, à $2000 \mathrm{~m}$ la température moyenne est $0^{\circ} \mathrm{C}$, de 1700 à $1800 \mathrm{~m}$ d'altitude elle arrive à $1,2^{\circ} \mathrm{C}$, tandis qu'à la périphérie des montagnes cette température augments jusqu'à $7^{\circ} \mathrm{C}$ sur le versant sud et jusqu'à $6^{0} \mathrm{C}$ sur le versant nordique (Buta et Buta, 1979).

Les précipitations varient aussi en fonction de l'altitude et de l'exposition. Ainsi, sur les versants de l'ouest et sud-ouest, à des altitudes qui dépassent $2200 \mathrm{~m}$, les quantités de précipitations annuelles dépassent $1400 \mathrm{~mm}$, tandis qu'à $1800 \mathrm{~m}$ d'altitude la moyenne annuelle est $1310 \mathrm{~mm}$ (Coldea, 1990).

\footnotetext{
${ }^{1}$ Université "Babeş-Bolyai", Faculté de Biologie et Géologie, 1, rue M. Kogălniceanu, 400084, Cluj-Napoca, e-mail: itantau@bioge.ubbcluj.ro
} 
Dans les Monts Rodnei, comme dans toutes les montagnes des Carpates roumaines, on constate une différenciation de la végétation en fonction de l'altitude, mais en corrélation avec les facteurs climatiques et édaphiques de la région. La végétation actuelle de la région a été étudiée assez récemment par Coldea (1990).

Pour mettre en évidence des nouveaux aspects de l'histoire de la végétation des Monts Rodnei nous avons abordé l'étude palynologique des deux petites tourbières situées à $1540 \mathrm{~m}$ d'altitude $\left(47^{\circ} 35^{\prime} \mathrm{N}, 2^{\circ} 49^{\prime} \mathrm{E}\right)$, dans les montagnes de Rodna (Fig. 1), sur la Vallée Bistricioara. Les tourbières se sont formées dans des dolines du massif calcaire de ces montagnes.

La végétation sur place est représentée par les associations de type SphagnoCaricetum rostratae et Caricetum limosae. La végétation limitrophe au site est représentée par les associations Soldanello majori- Piceetum et Swertio perennisCaricetum chordorrhizae (Coldea, 1990).

Les principaux taxons rencontrés à Ştiol sont: Pinus mugo, Picea abies, Sphagnum div. sp., Polytrichium commune, $P$. strictum, Eriophorum vaginatum, Carex rostrata, C. canescens, C. leporina, Nardus stricta, Deschampsia flexuosa, Alepecurus geniculatus, Luzula sudetica, Dryopteris carthusiana, Caltha laeta, Vaccinium myrtillus, V. vitis-idaea, Rhododendron ketschyi.

\section{METHODE DE TRAVAIL}

Les premières études dans la région ont été effectuées par Boşcaiu et al. (1983). Ils ont réalisé un sondage de $480 \mathrm{~cm}$ de profondeur, dans une tourbière installée dans une des dolines de Poiana Ştiol, sur la Valée Bistricioara. Les résultats palynologiques ont montré que la sédimentation de la tourbe a commencé pendant la phase à Pinus.

Nous avons réalisé 4 sondages, dans deux petites tourbières, à l'aide d'un carottier russe, manuel, de $8 \mathrm{~cm}$ de diamètre et un autre de $5 \mathrm{~cm}$ de diamètre. Seulement deux sondages ("Poiana Ştiol 1" et "Poiana Ştiol 4"), effectués dans une tourbière située dans une petite dépression, à coté d'une bergerie, sont étudiés dans cet ouvrage. Les sédiments les plus profonds ont été récoltés dans une argile grise. La description des carottes de Poiana Ştiol 1 et 4 est détaillée dans le Tableau 1.

Après l'analyse et la description lithologique des carottes nous avons prélevé des échantillons. Pour le traitement chimique des échantillons nous avons utilisé la méthode de la séparation par liqueur dense Thoulet (Goeury et Beaulieu, 1979).

Les déterminations polliniques ont été effectuées au niveau de famille, de genre ou de l'espèce s'il a été possible. Une moyenne de 250 grains de pollen d'arbres (AP $=$ arborum pollen) et le pollen des herbacées (NAP = non arborum pollen) correspondant ont été comptés pour chacun des préparâtes microscopiques.

Les pourcentages ont été calculés par le rapport de chaque taxon, à la somme totale du pollen des arbres, des herbacées et de quelques spores aussi. Nous avons déterminé également le rapport A.P./N.A.P. pour mieux illustrer le type d'écosystème dominant en fonction de la période du temps. Ce rapport apparaît sur les diagrammes polliniques (Fig. 2 et 3 ).

Pour obtenir la représentation graphique on a appelé à l'aide du logiciel GpalWin du Laboratoire de Botanique Historique et Palynologie de Marseille (Goeury, 1997). Sur les diagrammes polliniques les fréquences inférieures à $0,5 \%$ sont représentées par des points. 
La séquence Poiana Ştiol 1 (PS1) a fourni 55 spectres, celle de et celle de Poiana Ştiol 4 (PS4), 48 spectres. 85 taxons ont été déterminés en PS1 et 92 en PS4.

Dans les diagrammes polliniques (Fig. 2 et 3), les Cypéracées et les spores monolètes ont été exclues de la somme pollinique totale pour le calcul des fréquences relatives.

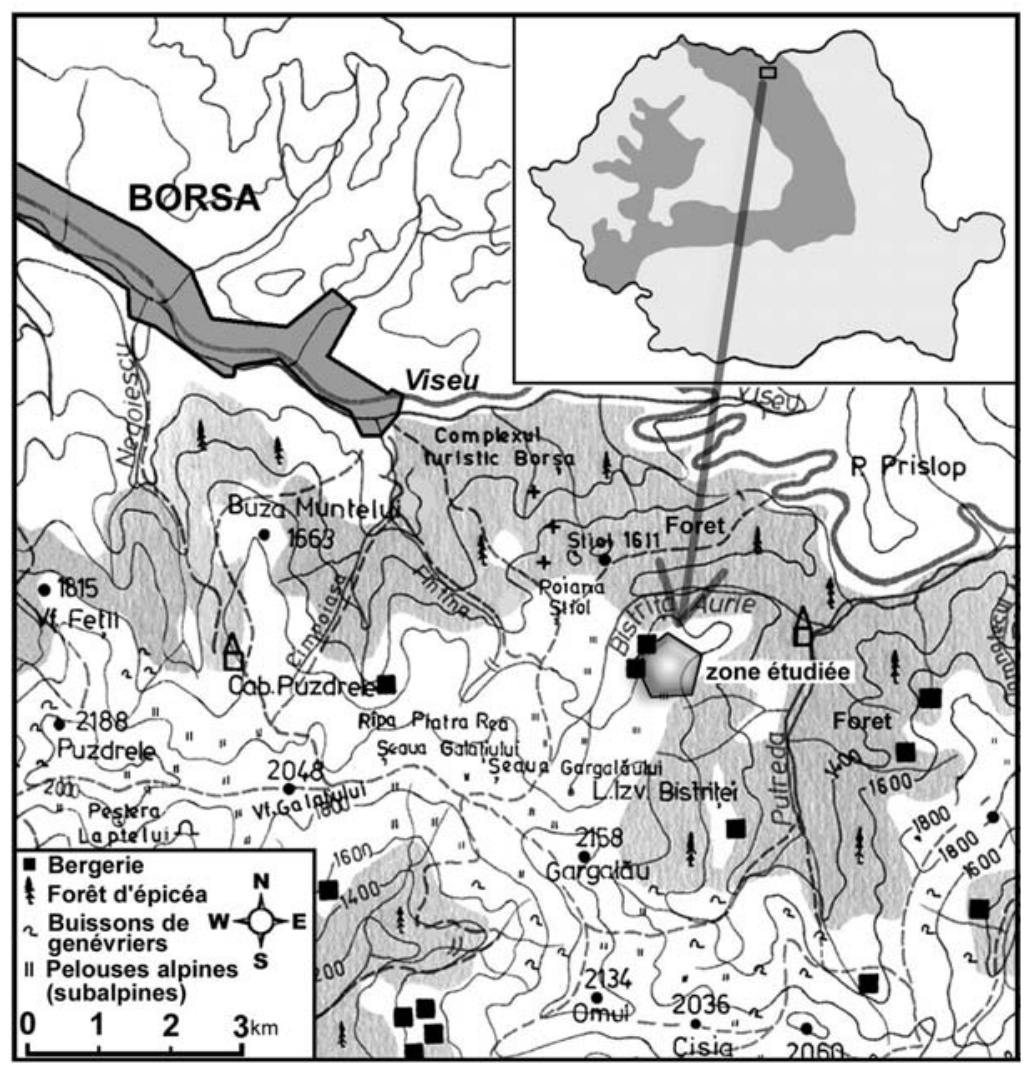

Fig. 1. Localisation de la zone étudiée.

\section{ANALYSE DES DONNEES}

Dans l'interprétation des résultats sporo-polliniques nous avons utilisé la méthode des zones polliniques (Birks, 1970, 1986).

Les 9 zones locales repérées sont comparées dans le Tableau 2; ce dernier suggère les remarques suivantes:

- les deux séquences débutent pendant l'Atlantique;

- les zones polliniques $\mathbf{a}, \mathbf{b}$ et $\mathbf{c}$ de la séquence PS4 s'ont l'équivalent d'une seule zone (b) de la séquence PS1;

- la végétation est dominée par Picea. 


\section{IOAN TANȚĂU}

\section{Zone locale 1}

C'est une zone présente en PS1. Elle est caractérisée par des taux d'Ulmus d'environ $5 \%$ et des taux élevés de Corylus et surtout de Picea.

\section{Zones locales 2, 3, et 4}

Elles sont caractérisées par l'extension de la courbe de Carpinus, des occurrences régulière de Fagus et des taux de Corylus d'environ $15-20 \%$.

Le pollen de Picea reste toujours à des taux élevés (50-60\%). On trouve quelques particularités dans la séquence PS4 (les zones PS4a et PS4c) où dans certains spectres, les taux de Carpinus ne dépassent pas $1 \%$.

\section{Zone locale 5}

Le début du Subboréal est marqué à Poiana Ştiol par un palier de Carpinus, dont la fréquence atteint $10 \%$ dans la séquence PS1 (zone PS1d); en PS4 (zone PS4d) elle ne dépasse pas $5 \%$. Dans cette zone les occurrences de Fagus sont régulières.

\section{Description des carottes de Poiana Ştiol 1 et 4}

Tableau 1.

\begin{tabular}{|cl|}
\hline ŞTIOL 1 & \\
$40-95 \mathrm{~cm}$ & Tourbe à Carex, peu évoluée, fibreuse \\
$95-165 \mathrm{~cm}$ & Tourbe à Carex-Sphagnum, peu évoluée, assez compacte, brune. \\
$165-280 \mathrm{~cm}$ & Tourbe à Carex-Sphagnum, évoluée, modérément humide, brune. \\
$280-295 \mathrm{~cm}$ & Tourbe à Carex-Sphagnum, évoluée, argileuse, brune. \\
$>295 \mathrm{~cm}$ & Argile grise \\
\hline ŞTIOL 4 & \\
$0-200 \mathrm{~cm}$ & Tourbe à Carex, peu évoluée, fibreuse, brune. \\
$200-242 \mathrm{~cm}$ & Transition vers gyttja grise \\
$242-250 \mathrm{~cm}$ & Gyttja argileuse grise \\
$250-286 \mathrm{~cm}$ & Gyttja noire \\
$>286 \mathrm{~cm}$ & Argile grise \\
\hline
\end{tabular}

\section{Zones locales 6}

Ce sont les zones où Carpinus atteint son maximum, même si dans les séquences PS1 (zone PS1d), et PS4 (zone PS4e), sa fréquence ne dépasse pas $10 \%$. Le début de la courbe de Fagus, la montée de Pinus, la régression d'Ulmus et des occurrences d'Abies constituent les autres faits marquants de cette zone.

\section{Zones locales 7 et 8}

Les phénomènes communs à ces zones sont un palier de Fagus à des taux élevés (10-25\%), la chute des taux de Carpinus et le début des présences continues d'Abies.

\section{Zone locale 9}

C'est la zone où Fagus est à son maximum (de $20 \%$ à $50 \%$ ); on note aussi la chute des taux de Picea et les premières occurrences de Juglans.

Les herbacées progressent et on remarque les premières notations de céréales (pro parte) et de Secale, indices d'une activité agricole dans la région. 


\section{CONCLUSIONS}

Les plus profonds sédiments récoltés sont une argile grise. Le début de la sédimentation de la tourbe s'est produit pendant l'Atlantique. La végétation de cette période est dominée par Picea, Corylus et Ulmus.

Picea est l'un des taxons très importants de la végétation holocène des Monts Rodnei. II connaît une nouvelle expansion, après celle survenue durant le Tardiglaciaire, des le début de l'Holocène, dans les Monts Retezat, dans les Mont Căliman (Fărcaş et al., 1999) et dans les Monts Gutâi (Björkman et al., 2002).

Tableau 2

Comparaison des zones polliniques des séquences de Poiana Ştiol 1 et 4

\begin{tabular}{|c|c|c|c|c|}
\hline 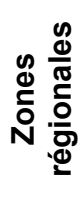 & 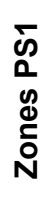 & $\begin{array}{l}\text { ஸे } \\
0 \\
\text { g } \\
\stackrel{0}{0}\end{array}$ & Caractéristiques du contenu pollinique & 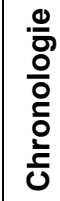 \\
\hline 9 & $\mathrm{~g}$ & $\mathrm{~h}$ & Taux maximums de Fagus et d'Abies. & \multirow[b]{2}{*}{ SA } \\
\hline 8 & $f$ & g & $\begin{array}{l}\text { Palier de Fagus à des taux élevés (10-20\%), chute des } \\
\text { taux de Carpinus. }\end{array}$ & \\
\hline 7 & e & $f$ & $\begin{array}{l}\text { Palier de Fagus, réduction de Carpinus. Queue de courbe } \\
\text { de Abies. }\end{array}$ & \multirow{3}{*}{ SE } \\
\hline 6 & $d$ & $\mathrm{e}$ & Maximum de Carpinus. Queue de courbe de Fagus. & \\
\hline 5 & C & $\mathrm{d}$ & Palier de Carpinus, occurrence de Fagus. & \\
\hline 4 & \multirow{3}{*}{ b } & $\mathrm{C}$ & Queue de courbe de Carpinus, occurrence de Fagus et & \multirow{4}{*}{ AT } \\
\hline 3 & & $\mathrm{~b}$ & Abies. & \\
\hline 2 & & a & Corylus à 15-20\%. Taux élevés de Picea. & \\
\hline 1 & $a$ & & Occurrences de Fagus. & \\
\hline
\end{tabular}

Le début de l'expansion de Carpinus, qui correspond à la fin de l'optimum de Corylus, s'est produit à la fin de l'Atlantique. Dans les Carpates roumaines l'expansion majeure du Carpinus est datée entre environ 6500 ans BP dans les Monts Semenic (Rosch et Fischer, 2000), à Tăul Zănoguții (Fărcaş et al., 1999) et dans les monts Apuseni (Bodnariuc et al., 2002), 4200 ans BP dans les Monts Rodnei (Tanțău, 2003; Tanțău et Fărcaş, 2004) et 4000 ans BP à Şteregoiu (Feurdean et al., 2001; Björkman et al., 2002). II semble avoir une progression du sud vers le nord de ce taxon.

L'expansion des forêts dominées par Fagus est beaucoup plus tardive en Roumanie par rapport aux pays de l'Europe de l'Ouest et de la Péninsule Balkanique. Elle est datée généralement des alentours de 4000 ans BP, comme dans la partie sud-est de Pologne (Ralska-Jasiewiczowa et Latalowa, 1996). Des données récentes montrent un âge d'environ 5600 ans BP pour l'expansion de Fagus à Ic Ponor, dans les Monts Apuseni (Bodnariuc, 2000; Bodnariuc et al., 2002). Dans les Monts Rodnei l'événement c'est produit vers 3500 ans BP (Tanțău, 2003; Tanțău et Fărcaş, 2004). 
IOAN TANȚĂU

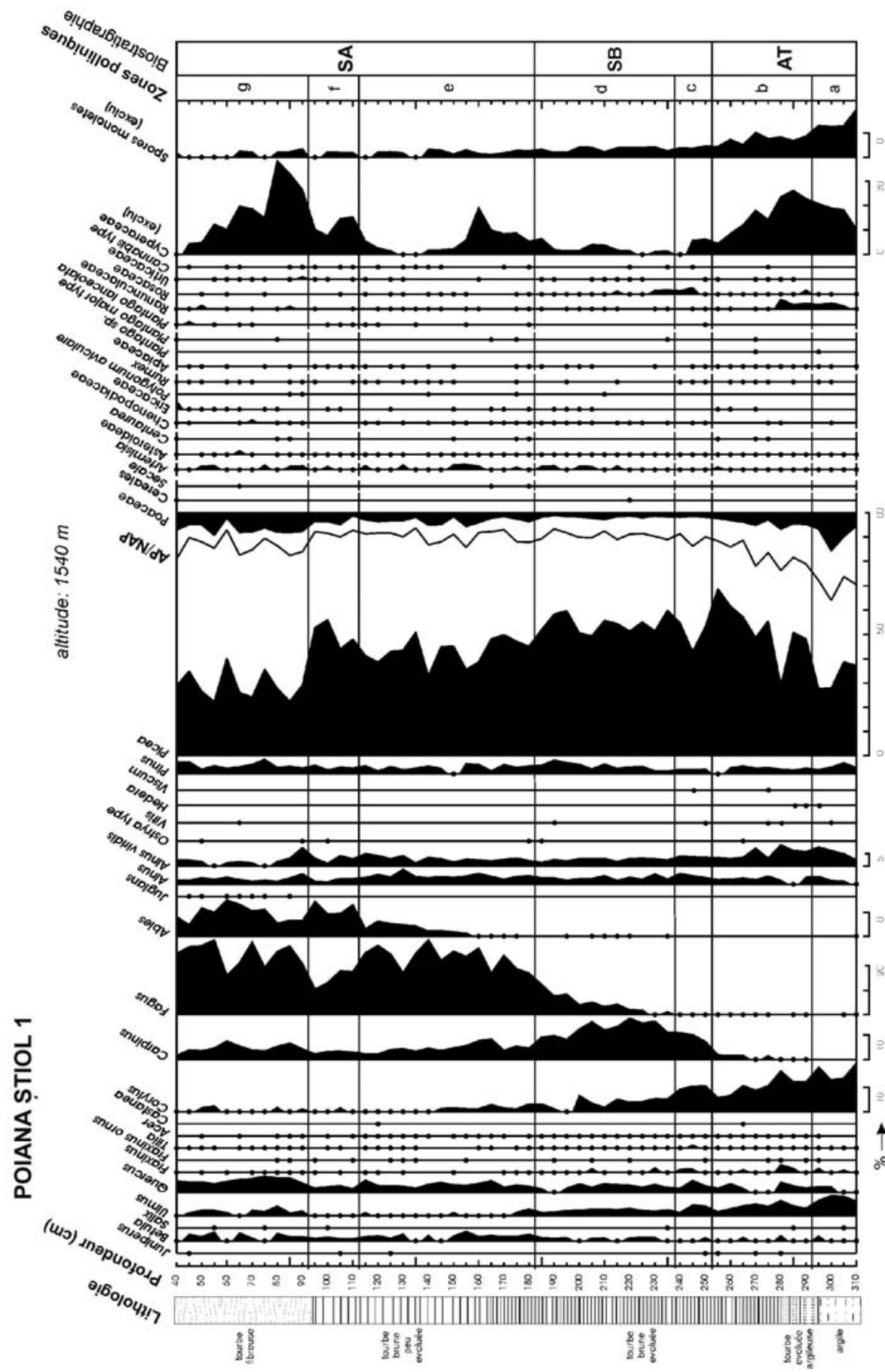

Fig. 2. Diagramme pollinique simplifié de Poiana Ştiol 1. 
NOUVELLES CONTRIBUTIONS A LA CONNAISANCE DE L'HISTOIRE DE LA VEGETATION ...

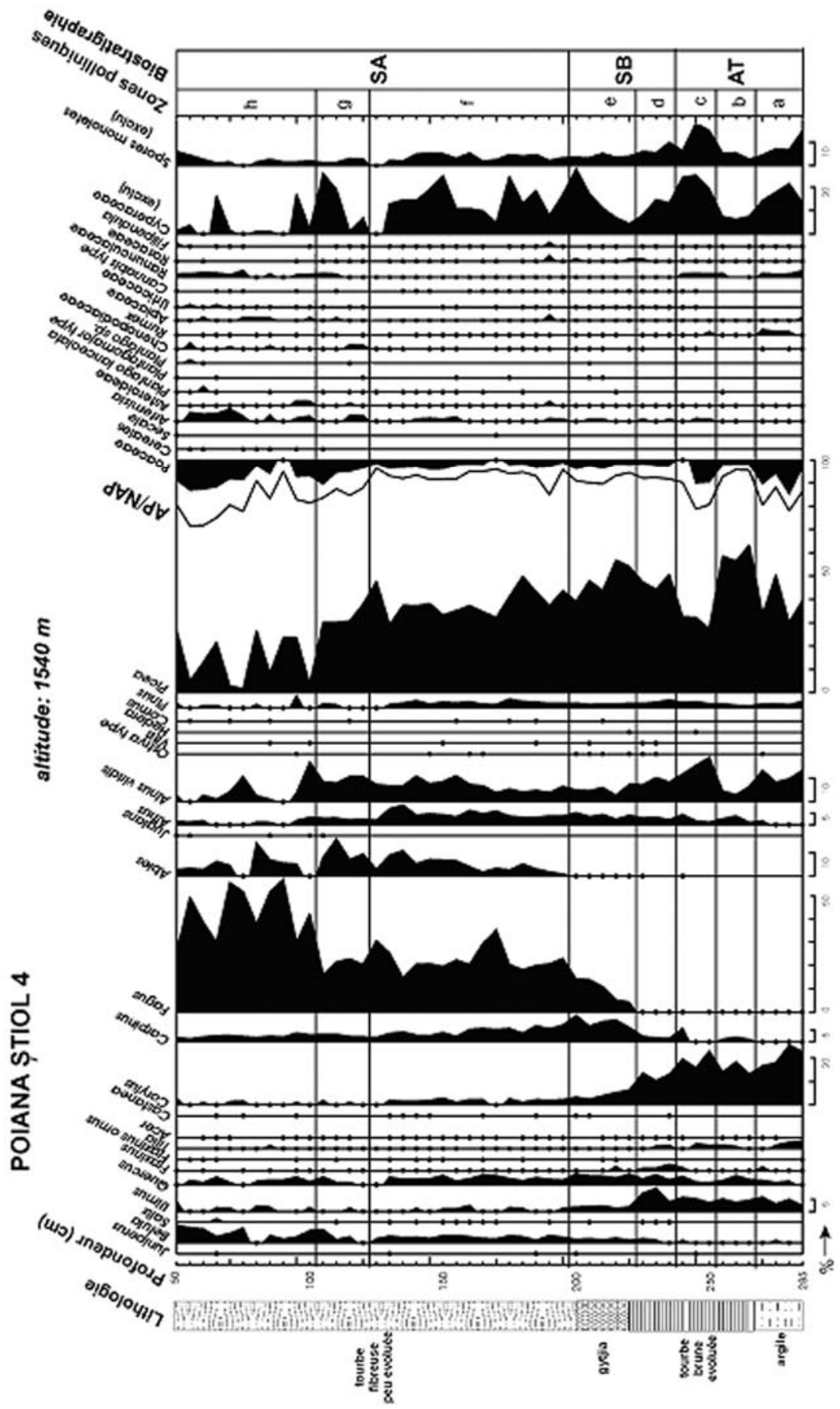

Fig. 3. Diagramme pollinique simplifié de Poiana Ştiol 4. 


\section{IOAN TANȚĂU}

Toutes les données suggèrent que l'expansion de Fagus soit produite à partir des populations situées dans la partie sud et ouest de l'arc carpatique.

Les études palynologiques de Roumanie montrent l'installation très tardive du sapin dans les structures forestières holocène, pendant le Subboréal. Elle est datée vers 4100 ans BP dans les Monts Semenic (Rösch et Fischer, 2000) et beaucoup plus tard (vers 2000 ans BP) dans les Monts Rodnei (Tanțău, 2003; Tanțău et Fărcaş, 2004).

Les premières occurrences de céréales apparaissent très tardivement à Poiana Ştiol par rapport aux autres zones de Roumanie (Tanțău, 2003).

La dernière partie du Subatlantique est la période de l'optimum pour les cultures et pour le pâturage. L'activité humaine est attestée par la présence du pollen de céréales indéterminées, Secale, Plantago lanceolata et Rumex.

\section{BIBLIOGRAPHIE}

Björkman S., Feurdean A., Cinthio K., Wohlfarth B., Possnert G., 2002 Late glacial and early Holocene vegetation development in the Gutâiului Mountains, northwestern Romania. Quaternary Science Reviews 21:1039-1059.

Bodnariuc A., 2000, Histoire holocène de la végétation des Monts Apuseni (Carpates Occidentales, Roumanie) - étude palynologique. Thèse de doctorat, Univ. Paul Sabatier Toulouse III, Lab. d'Ecologie Terrestre.

Bodnariuc A., Bouchette A., Dedoubat JJ, Otto T., Fontugne M., Jalut G., 2002, Holocene vegetational history of the Apuseni mountains, central Romania. Quaternary Science Reviews 21:1465-1488.

Birks H.J.B., (1974), Numerical zonation of Flandrian pollen data. New Phytol. 73:351-358.

Birks H.J.B., 1986, Numerical zonation, comparison and correlation of Quaternary pollen-stratigraphical data. In: Berglund, B.E. (ed.): Handbook of Holocen paleoecology and paleohydrology, Chicester, p. 743-774.

Boşcaiu N., Lupşa V., Oros E., Pînzaru Ghe., 1983, Aspecte din trecutul vegetației munților Rodnei. In: Rezervatia naturală Pietrosul Rodnei la 50 de ani de la înființare. Lucr. Simp. Aniv., 2-4 sept. 1982, Cluj-Napoca, Academia RSR, Fil. Cluj-Napoca, p. 232-249

Buta I., Buta A.A., 1979, Munții Rodnei, Colecția "Munții noştri", Bucureşti.

Coldea Gh., 1990, Muntii Rodnei - Studiu geobotanic. Ed. Acad. Române, Bucureşti, 183 p.

Ciobanu I., Diaconeasa B., Suteu S., 1969, Analize de polen din unele mlaştini de turbă din zona estică a masivului Rodnei. Contribuții Botanice, Cluj-Napoca, p. 301-305.

Ciobanu I., Diaconeasa B., Suteu S., 1969, Analize de polen din unele mlaştini de turbă din zona sudică a masivului Rodnei. Contribuții Botanice, Cluj-Napoca, p. 37-43.

Fărcaş S., Beaulieu JL de, Reille M., Coldea G., Diaconeasa B., Goeury C., Goslar T., Jull T., 1999, First ${ }^{14} \mathrm{C}$ datings of late Glacial and Holocene pollen sequences from Romanian Carpathes. Comptes Rendus de l'Académie des Sciences de Paris, 322:799-807.

Feurdean A., Björkman L., Wohlfarth B., 2001, A Paleoecological Reconstruction of the Late Glacial and Holocene based in multidisciplinary studies at Steregoiu site (Gutai Mts., NW Romania). Studia Universitatis Babes-Bolyai, Geologia, 46(2), 125-140. 
NOUVELLES CONTRIBUTIONS A LA CONNAISANCE DE L'HISTOIRE DE LA VEGETATION ...

Goeury C., 1997, GpalWin: gestion, traitement et représentation de la paléoécologie. În: XV-ème Symp. de l'A.P.L.F., sept. 1997, Lyon, p.31.

Goeury C., Beaulieu J.-L. de, 1979, A propos de la concentration du pollen à l'aide de la liqueur de Thoulet dans les sédiments minéraux, Pollen et Spores, 21, 1-2, p. 239-251, Paris.

Rösch M., Fischer E., 2000, A radiocarbon dated Holocene pollen profile from the Banat mountains (Southwestern Carpathians, Romania). Flora 195: 277-286.

Tanțău, I., Fărcaş, S., 2002, Aspects de l'histoire de la vegetation holocene des Monts Rodnei (Carpates Orientales). Studia Univ. Babeş-Bolyai, Geologia, Special Issue 1, p. 331-339, ClujNapoca.

Tanțău, I., 2003, Recherches pollenanalytiques dans les Carpates Orientales (Roumanie). Histoires de la végétation et de l'impact humain. Thèse de doctorat, Universités Aix-Marseille III et Babeş-Bolyai, Cluj-Napoca.

Tanțău, I., Fărcaş, S., 2004. Chronologie de l'histoire de la vegetation holocène de Monts Rodnei (Carpates Orientales). Contribuții Botanice (sous press). 